\title{
A New Computational Model of Friction Applied to Haptic Rendering
}

\author{
Vincent Hayward \\ Center for Intelligent Machines \\ McGill University, 3480 University Street \\ Montreal, Qc H3A 2A7, Canada \\ hayward@cim.mcgill.ca \\ Brian Armstrong \\ Department of Electrical Engineering and Computer Science \\ University of Wisconsin - Milwaukee, Post Office Box 784 \\ Milwaukee, Wisconsin 53201-0784, U.S.A. \\ bsra@csd.uwm.edu
}

\begin{abstract}
A time-free, drift-free, multi-dimensional model of friction is introduced. A discrete implementation is developed which exhibits four solution regimes: sticking, creeping, oscillating, and sliding. Its computational solution is efficient to compute online and is robust to noise. It is applied to haptic rendering.
\end{abstract}

\section{Introduction}

Friction occurs almost everywhere. Many things, including human acts, depend on it. It is almost always present in machines. Usually friction is not wanted, so a great deal has been done to reduce it by design, or by control. In the present case, we want to synthesize it, so it can be presented under computer control to a subject using a haptic device [1]. The model introduced in this paper is also a possible contribution to the existing model-based compensation techniques. The model has the following properties:

1. It is time free (autonomous), only displacements enter in the formulation.

2. It neither drifts, nor relaxes.

3. It is robust to noise, input is not assumed to be noise-free.

4. A discrete formulation exists which is online and computationally efficient.

5. It has four regimes, one of them is a quasi-periodic oscillation.

6. It accounts for vector motions and forces (2D or $3 \mathrm{D})$.

7. Its parameters have a simple physical interpretation.

8. It has a continuous counterpart.

\section{Previous Models}

Friction refers to the production of force as a result of relative movement between two objects in contact. The force must oppose motion when there is sliding (i.e. macroscopic motion). This forms the physical basis of dissipation. When there is adherence, microscopic motions can arise with contact compliance. To good approximation, the contact force must balance the net external forces. The force of adhesion in is not literally friction, but must be considered 
as part of a static friction model. Below a threshold of micro-displacement (or equivalently of force), the net macroscopic motion is truly identically zero. This forms the basis of energy storage. We can then speak of several states: relaxed, tense adhesion, and sliding. This basic model can be enhanced with the addition of dynamic friction effects which depend on time (rising static friction, frictional memory), or on state (friction irregularities, or dependencies as a function of space or of velocity as in hydrodynamic dependencies). For a complete survey, see [2]. In this paper, these model enhancements are not considered.

\subsection{Presliding Displacement and Static Friction}

Accounting for static friction and the transition to sliding motion is a considerable challenge for friction models, both in terms of the surface physics - the origin of adhesion was long debated and still cannot be predicted accuratelyand in terms of modeling and simulation. Bodies in contact exhibit "presliding displacement" even when there is no true sliding. This motion arises with surface deformation in the contact. Over a small elastic regime energy is stored and a mass-spring behavior can be observed [3].

Dahl was the first to systematically study and model presliding displacement [4]. His model is further described below. A point addressed here is that Dahl's model, and more recent models based on it, exhibits drift when subjected to an arbitrarily small bias force and arbitrarily small vibrations. This drift is spurious: objects set on a slope and subjected to small vibrations do not continuously creep down the slope. Drift in the friction model is important for haptic interfaces because small vibrations originate from involuntary hand tremors, even in healthy individuals.

To define terms for modeling presliding displacement, consider first the one dimensional case of two objects with point contact, see the figure below. One object is termed the fixed object, the other is the moving object.

Friction is best understood by considering two

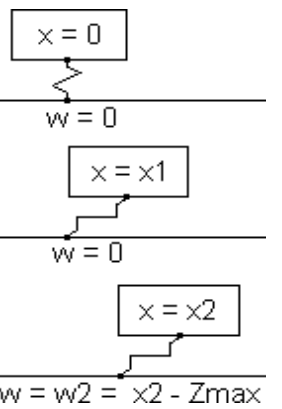
points. One of them belongs to the moving object, call it $x$. The other defines an adhesion point, call it $w$. During adhesion, $w$ is attached to the fixed object, so $z=x-w$, a signed quantity, describes micromovements between the two objects. Most models, including Dahl's, define the friction force to be proportional to the strain $z$ as if the two objects were attached by a spring. The quantity $|z|$ is not allowed to exceed a small value called the breakaway distance (equivalently, the breakaway force), that we call here $z_{\max }>0$.

When $|z|$ reaches $z_{\max }$ while $x$ monotonically increases (or decreases), the contact becomes fully tense and $w$ relocates so that at all times $|z|=z_{\text {max }}$. While the contact is fully tense, necessarily $\dot{x}=\dot{w}$ and $\dot{z}=0$. This is called sliding. The magnitude of the friction force varies with the normal force, but this relationship is not considered here. 


\subsection{Computational Models}

Karnopp considers computer simulations of friction using a model that switches from one dynamic equation to another [5]. It has been applied by Salcudean and Vlaar to haptic rendering [6]. It is similar in concept to the model just described but must use a velocity threshold to switch from sliding to adhesion. When sliding, the friction force is replaced by a viscous force.

Haessig and Friedland, discussing the original Dahl model, develop computational versions including a "bristle model" and a "reset integrator model" [7]. The bristle model has been applied to haptics by Chen et al. [8].

\subsection{Dahl's Model}

Dahl's formulation may be viewed as an attempt to account for the conceptual model of Section 2.1 using one single differential equation. The more general form proposed by Dahl is as follows $[9,10]$ :

$$
\dot{f}=\sigma_{0} v\left|1-f / f_{c} \operatorname{sgn} v\right|^{i} \operatorname{sgn}\left(1-f / f_{c} \operatorname{sgn} v\right),
$$

where $v=\dot{x}=d x / d t, f$ is the friction force, $f_{c}$ the Coulomb force and $\sigma_{0}$ the assumed stiffness relating force to strain. The model may be formulated in terms of displacements, posing $f=\sigma_{0} z, f_{c}=\sigma_{0} z_{\max }$, and $\alpha=1 / z_{\max }$ :

$$
\dot{z}=v|1-\alpha \operatorname{sgn} v z|^{i} \operatorname{sgn}(1-\alpha \operatorname{sgn} v z) .
$$

This expression conveys that the rate of change of $z$ is zero when (i) $v$ is zero or when (ii) $v$ and $z= \pm z_{\max }$ have the same signs. In both cases the contact remains tense, otherwise $|z|$ must decrease or increase so that $|z|$ can never exceed $z_{\max }$. The exponent $i$ expresses how forcefully $z$ changes when $v$ is not zero and $|z|<z_{\max }$. The $|$.$| and sign parts ensure the same behavior for any$ integer value of $i$. Many model enhancements introduce a dependency of $\alpha$ on time or state, as proposed by Canudas et al. [10].

From now on, we take $i=1$ without affecting the discussion:

$$
\dot{z}=v(1-\alpha \operatorname{sgn} v z)=\dot{x}-\dot{w} .
$$

Figure 1 shows $x(t)$ for a system governed by $\ddot{x}+\dot{x}=0.5+0.1 \cos t-f(t)$, when $f(t)$ is given by Eq. (1), with $\sigma_{0}=100$ and $f_{c}=1$.

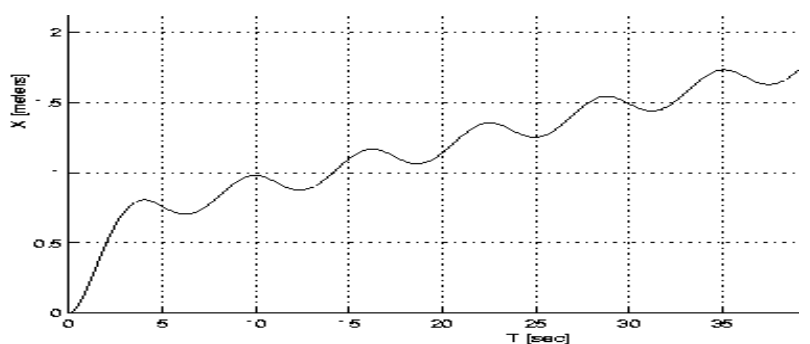

Figure 1. Solution obtained with Matlab ${ }^{T M}$ ODE solver

Modeling the contact as a compliance should yield an oscillation for $x(t)$ as a filtered version of $f(t)$, since the applied force lies in the range $[0.4,0.6]$, but a net average velocity of 0.03 meters per second is observed, although the breakaway force, or Coulomb friction level, is never applied. 


\subsubsection{Behavior at reversals}

Of particular interest is the behavior of the solution at reversals, that is when $v$ changes sign. Because of the compliance in the contact, external rapidly varying applied forces will result in reversals of the microscopic motion.

To gain further insight, it is useful to rewrite the model in autonomous form, bearing in mind that in our conceptual account (Section 2.1), time was never needed, except to express that when there is sliding $\dot{x}=\dot{w}$ and $\dot{z}=0$. The same idea can be expressed without time by writing that during sliding $x=w \pm z_{\max }$ and $|z|=z_{\max }$. The corresponding autonomous equation is obtained by eliminating time from Dahl's model:

$$
d z / d x=1-\alpha \operatorname{sgn} d x z
$$

thinking of $z$ as a function of $x$ instead of $t$. It is now easy to find the possible values of $d z / d x$. For a tense contact, this quantity has a value zero when $d x$ and $z$ have the same sign (as expected) and 2 when they have opposite signs.

\subsubsection{The drift and relaxation ladders}

When $z$ and $d x$ have opposite signs, $z$ changes up to twice as fast as $x$ does. In Section 2.1, we imagined $z$ to change at most at the rate of $x$, to keep $w$ invariant. Consider first that $x$ oscillates between two values. In Figure 2(a) the plot of $z$ against $x$ is shown, for all possible paths of $z(x)$ when $x$ varies between values further apart than $2 z_{\max }$. $z(x)$ traces closed major hysteresis loops which return to the point that they left since the ascending and the descending branches are symmetrical: we may substitute $z$ by $-z$ and $d x$ by $-d x$ leaving Eq. (2) invariant (a symmetry group). However, for small cycles around a non null value of $z$, the symmetry is broken: substitute $z$ by $z-a$ and Eq. (2) no longer is invariant. The minor paths no longer are symmetrical, they do not even trace loops but ladders, see Figure 2(b)(c), as shown now.

Suppose that $x$ cycles with a small amplitude $n$ around any location in the phase portrait. Call $\left\{z_{m}\right\}$ the sequence of extrema of $z$ at each reversal; $\Delta z^{+}$ and $\Delta z^{-}$the increase of $z$ on the ascending and descending paths respectively.

$$
\left.\begin{array}{l}
d x>0: \Delta z^{+}=n(+1-\alpha z) \\
d x<0: \Delta z^{-}=n(-1-\alpha z)
\end{array}\right\} \frac{\Delta z}{z}=-2 \alpha n .
$$

$\left\{z_{m}\right\}$ converges (relaxes) to zero geometrically as in Figure 2(b). Now, an external force causing $z$ to oscillate around a nominal value yields a diverging drift ladder for $x$. Call $m$ the amplitude of the oscillation of $z$ around $z_{n o m}$. Posing $\beta=\alpha z_{\text {nom }}<1$, we find the progress of $x$ between two like signed extrema of $z$ to be: $2 m \beta /\left(1-\beta^{2}\right)$, see Figure $2(\mathrm{c})$. This is contrary to physical evidence. For small perturbations (say Brownian motion, permanent small tremors), a frictional contact neither relaxes nor drifts.

A drift-free friction model could either (i) have symmetrical minor hysteresis loops (this might not be easy to derive) or (ii) have no minor paths. In other terms, the ascending branches should overlap the descending ones to preserve symmetry. Memory would then be solely encoded in the sliding regime as in Figure 2(d). For the purpose of this paper, we opt for the second possibility. 


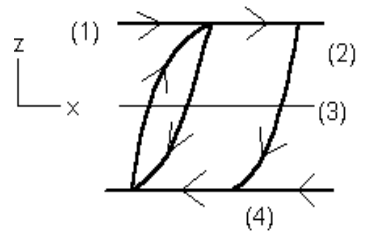

(a)

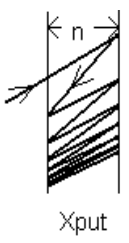

(b)

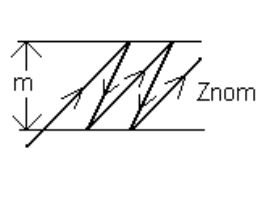

(c)

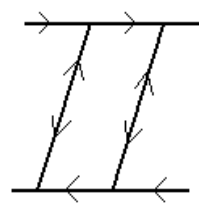

(d)

Figure 2. (a) Major hysteresis loops traced by Dahl's model. (1) sliding: $d z / d x=0: d x>0, z>0,(2)$ start relaxing: $d z / d x=2: d x<0, z>0,(3)$ relaxed $d z / d x=1: d x<0, z=0$, (4) finish tensing $d z / d x=0: d x<0, z<0$. (b) Converging drift ladder for small oscillations of $x$ around $x_{p u t}$. (c) Diverging ladder for small oscillations of $z$ around $z_{n o m}$. (c) Drift free friction.

We now depart from the Dahl model, making $\alpha$ depend on $z$ :

$$
d z / d x=1-\alpha(z) \operatorname{sgn} d x z .
$$

We call $\alpha(z)$ the adhesion map. It is a nonlinear map which controls the rate of change of $z$, so that $w$ is allowed to move only when the contact is sufficiently tense. In general, $\alpha(z)$ must be identically zero for a range of values around the origin and have two asymptotes at $\alpha(z)=1 / z_{\max }$ for large values of $z$. There are many possible choices for $\alpha(z)$, we explore two in Section 3.2.

\section{An Online Discrete Scalar Model}

The object is to compute a sequence $\left\{z_{k}\right\}$ given a sequence of position measurements $\left\{\bar{x}_{k}\right\}$. We first integrate (3) with respect to $x$ over an interval $h$ of any length between two samples:

$$
z=x-\int_{h} \alpha(z) z|d x|=x-w
$$

A sampled version does not require a clock, samples may arrive irregularly.

$$
z_{k}=\bar{x}_{k}-w_{k} .
$$

Tension at time $k$ is the measured position $\bar{x}_{k}$, the counterpart of the first term of the continuous equation (4), minus the discrete version of $w$. The $w_{k}$ 's are the successive positions of the adhesion point: $w_{k}=w_{k-1}+\Delta w_{k} . \Delta w_{k}$ is the discrete counterpart of the integrand. Since samples are not necessarily equally spaced in time, simple filtering methods like discarding samples until a detectable displacement is found can be applied. Call $\bar{y}_{k}=\left|\overline{x_{k}-x_{k-1}}\right|$ to emphasize the fact that the magnitude of small movements can be detected separately from the position measurements $\bar{x}_{k}$.

$$
\Delta w_{k}=\alpha\left(z_{k}\right) \bar{y}_{k} z_{k} .
$$

\subsection{Stick-Slide}

We may compute $z_{k}$ based on the desired properties of the solution.

$$
w_{k}= \begin{cases}\bar{x}_{k} \pm z_{\max }, & \text { if }\left|\bar{x}_{k}-w_{k-1}\right|>z_{\max } ; \\ w_{k-1}, & \text { otherwise. }\end{cases}
$$


No integration is needed because we simply chose $\alpha(z)=0$ for $|z| \leq z_{\max }$ and $\alpha(z)=1 / z_{\max }$ elsewhere. Note that we can choose $z_{\max }$ to be very small, at the resolution limit of the position measurements. Calculations are indeed minimal. This formulation exhibits two regimes: stuck and sliding.

This model can be viewed as a filter which responds as shown on Figure $2(\mathrm{~d})$. It can be used to detect extrema and signs of the derivative of $x$, robustly with respect to noise without ever estimating a derivative.

\subsection{A More General Model}

In an effort to find solutions for $z(x)$ which exhibit richer behaviors, we no longer take the computational shortcut of the previous section. The sequence of $\left\{w_{k}\right\}$ is now obtained from explicit Euler integration summing a sequence of estimated incremental displacements.

$$
w_{k}=\left\{\begin{array}{lr}
\bar{x}_{k} \pm z_{\max }, & \text { if } \alpha\left(\bar{x}_{k}-w_{k-1}\right)\left|\bar{x}_{k}-w_{k-1}\right|>1 \\
w_{k-1}+\bar{y}_{k} \alpha\left(\bar{x}_{k}-w_{k-1}\right)\left(\bar{x}_{k}-w_{k-1}\right) & \text { otherwise }
\end{array}\right.
$$

\subsubsection{Stick-slip-slide}

We now take $\alpha(z)$ to be 0 for $|z| \leq z_{\text {stick }}$ and $\alpha(z)=1 / z_{\max }$ elsewhere. The parameter $z_{\text {stick }}$ may be chosen to be equal to $z_{\text {max }}$ in which case this more elaborate model reverts to that of the previous section. Now, consider that $z_{\text {stick }}$ is larger than $z_{\max }$. When $z$ is below $z_{\max }$, the model behaves like in the previous section and does not drift. However, if the external force is sufficient for $z$ to reach $z_{\text {stick }}, w$ slips, the contact becomes stuck and the cycle starts again, yielding relaxation oscillations akin to a stick-slip behavior. Moreover, if the motion is sufficiently rapid, $z$ stays at $z_{\max }$ and the oscillations vanish, yielding a sliding regime.

The sliding regime is entered and sustained when the condition $\alpha\left(z_{k}\right) z_{k}>1$ is met. It is therefore dependent on sampling rate since $z_{k}=\bar{x}_{k}-w_{k}$ depends on the interval between two samples. This means that the higher the sampling rate, the "tighter" a contact can be simulated (further discussed in 3.2.3).

\subsubsection{Stick-creep-slip-slide}

We may instead choose a smooth version of $\alpha(z)$, for example:

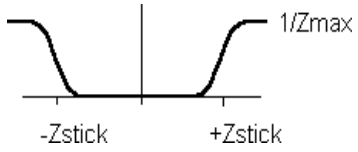

$$
\alpha(z)=\frac{1}{z_{\max }} \frac{z^{8}}{z_{\text {stick }}^{8}+z^{8}}
$$

which is nearly identical to zero for $\left|\left(z / z_{\text {stick }}\right)\right|<0.5$.

When the strain is sufficiently high, the model simulates an additional pre-sliding behavior because the model has a drift component (see discussion in [2], section 2.1.b.i). Let us call that creep to distinguish it from drift. If the motion of $x$ is sufficiently slow with respect to the drift and relaxation rates, the drift component consumes all of it so that $z$ remains below $z_{\text {stick }}$. On the other hand, if the motion of $x$ exceeds the drift rate, $z$ increases until it reaches $z_{\text {stick }}$. At this point, the behavior is the same as in the previous section. In summary, we have: stick, creep, oscillatory, and sliding regimes. See Figure 3. 


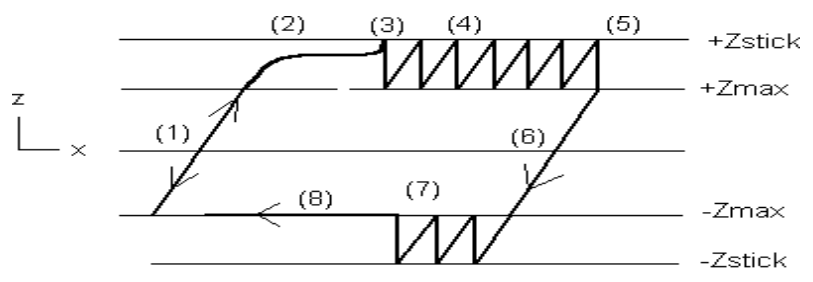

Figure 3. Trajectories when $z_{\max }<z_{\text {stick }}$. Labels: (1) stuck, (2) slow motion, the model creeps along, (3) a faster motion, $z$ changes like $x$ does minus the creep, (4) oscillatory regime, (5) motion reversal, (7) motion cannot be fast immediately after a reversal, (8) fast motions slide.

\subsubsection{Discussion}

The reader will notice that implicitly, time was reintroduced in the discussion. The rates are function of time: the drift and relaxation rates depends on noise (which in turn depend on time), the motion rate, the rate of the unforced dynamics of the system during the return to $z_{\max }$, and the sampling rate.

Additional care must be taken to determine the regime transition to sliding when $z$ is near $z_{\max }$ and to determine the creep rate when $z$ is near $z_{\text {stick }}$, independently from the sampling rate. It is a balance between these rates that determines the regime, but each regime is time-free.

Convergence is guaranteed for any value of $\alpha$ and any value of $\bar{y}_{k}$ since $z_{k}=x_{k}-w_{k}$ is never allowed to exceed $z_{\text {stick }}$.

\section{A Discrete Vectorial Model}

We now turn our attention to a multi-dimension friction model. Points $x$ and $w$ are now associated to two or three coordinates, we denote them $X$ and $W$; $z$ is now a vector $Z=X-W$. In 3 -D, we may think of $X$ as a particle and $W$ as the contact point with the ambient milieu.

It is helpful to look at Figure 4 to imagine the 2D counterpart of the scalar model just described.

(a)

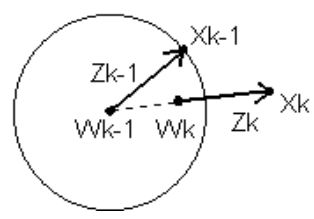

(b)

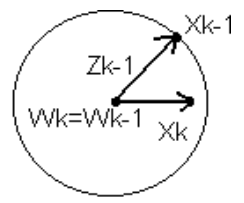

Figure 4. (a) Sliding: $X$ and $W$ move. (b) Stuck: $X$ moves, $W$ is invariant.

\subsection{A Simple Model}

The counterpart of (5) is straightforward since there is no integration:

$$
W_{k}= \begin{cases}\bar{X}_{k}-\frac{\bar{X}_{k}-W_{k-1}}{\left|\bar{X}_{k}-W_{k-1}\right|} z_{\max }, & \text { if }\left|\bar{X}_{k}-W_{k-1}\right|>z_{\max } \\ W_{k-1}, & \text { otherwise }\end{cases}
$$


This implements a robust motion direction detector, whereas the scalar version implements a sign detector. Since it is time-free, it is applicable to event-based input devices.

\subsection{A More General Model}

The most recent measurement $\bar{X}_{k}$ indicates the direction of movement, and $\bar{Y}_{k}=\left|\overline{X_{k}-X_{k-1}}\right|$. There are two choices for performing the integration.

$$
\begin{aligned}
\Delta W_{k} & =\alpha \bar{Y}_{k} Z_{k-1} & Z_{k} & =\bar{X}_{k}-W_{k-1} \\
Z_{k} & =\bar{X}_{k}-W_{k} & \Delta W_{k} & =\alpha \bar{Y}_{k} Z_{k}
\end{aligned}
$$

The second form is the only one that converges: the direction must be updated before the progress of $W$ (in the scalar case, the choice does not matter). This gives rise to the following discrete update law:

$$
W_{k}=\left\{\begin{array}{lr}
\bar{X}_{k}-\frac{\bar{X}_{k}-W_{k-1}}{\left|\bar{X}_{k}-W_{k-1}\right|} z_{\max }, \quad \text { if } \alpha\left(\bar{X}_{k}-W_{k-1}\right)\left|\bar{X}_{k}-W_{k-1}\right|>1 \\
W_{k-1}+\bar{Y}_{k} \alpha\left(\bar{X}_{k}-W_{k-1}\right)\left(\bar{X}_{k}-W_{k-1}\right), & \text { otherwise }
\end{array}\right.
$$

\section{Experimental Results}

Experiment were performed using the PenCat ${ }^{\mathrm{TM}}$ haptic device marketed by Haptic Technologies Inc. ${ }^{1}$ It is hooked up to a host personal computer via a serial line which permits update rates up to $400 \mathrm{~Hz}$, thanks to an embedded microprocessor.

In a first experiment, the friction model of Section 4.1 is used The thick line shows the trace of the "virtual pointer" on the screen as guided by the subject's hand, see Figure 5. The set of straight lines show $Z$ every $100 \mathrm{~ms}$. In (1) and (2), $Z$ is in the direction of motion and approximates closely the tangent to the trace. In (3), the subject's hand applies a force low enough so that adhesion occurs, and $W$ becomes stationary. $Z$ is no longer tangent to the trace (which is a point) but is guided through a $360^{\circ}$ counterclockwise sweep. In (4), motion stops also but $Z$ sweeps $90^{\circ}$ clockwise before the motion resumes. The same maneuver is carried out in (5), counterclockwise this time. The experiment terminates in (6), where the pointer is driven around a fixed point. To indicate how slow the movement is, consider that the time of travel from (1) to (2) is about 2 seconds (the figure is to scale 1:1).

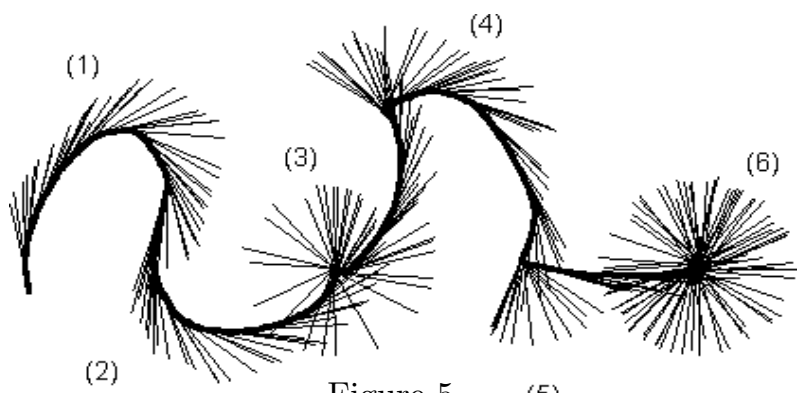

Figure 5. (5)

\footnotetext{
${ }^{1}$ www.haptech.com
} 
Here, the more general model of Section 4.2 is exemplified with $\alpha(z)$ as in Section 3.2.2. Figure 6 show the plot of $Z\left(z_{\max } \pm .25 \mathrm{~mm}, z_{\text {stick }} \pm .5 \mathrm{~mm}\right)$ against $X( \pm 50 \mathrm{~mm})$. The experiment starts in $(0)$ where the virtual pointer is moved slowly to the right. It traverses the stuck zone (1), and continues in the creep zone before converging in (2) to an equilibrium between the motion rate and the creep rate. In (3), the motion is reversed over a small distance and so the ascending branch retraces exactly the descending one. In (4), the motion is reversed over a larger distance, so the model creeps for a while in the negative direction, eventually forming a hysteresis loop. Shortly after, a larger force is applied so $z$ climbs to reach $z_{\text {stick }}$, then returns to $z_{\text {max }}$. Because the "feed-rate" is high enough, $z$ quickly meets $z_{\text {stick }}$ again, yielding a limit cycle (5). In (6), the motion is reversed, attempting to move to the left now at higher speed. The subject's hand takes a while to reach a sufficient speed so an oscillatory regime (7) is entered before sliding in (8).

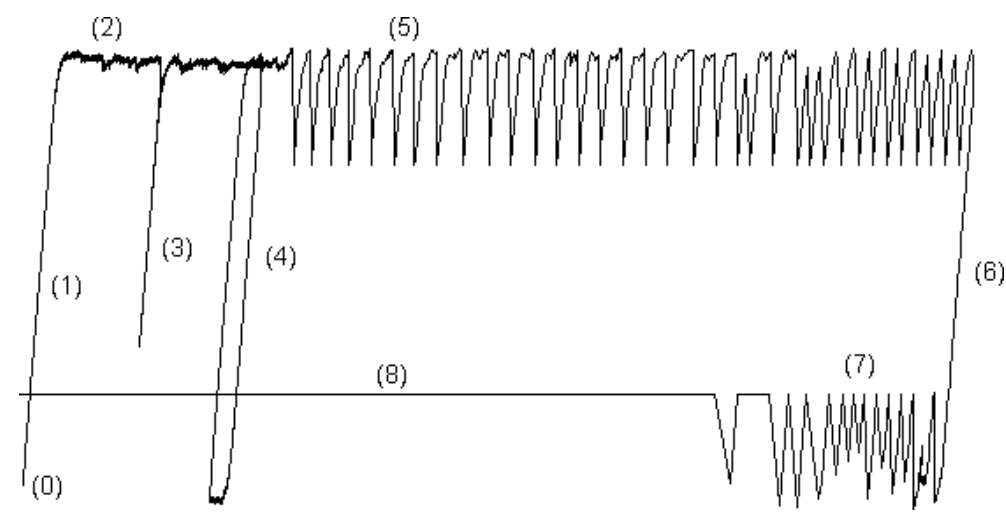

Figure 6.

Synthesized signals of this type of experiments have been recorded and are made available at www.cim.mcgill.ca/ haptic/squeaks.html.

\section{Conclusion}

It is shown that the Dahl friction model drifts when a small bias force is present with small oscillations of position. We hypothesize that this drift has not previously been identified because, in simulations, velocity during static friction can be exactly zero. In implementations where input is derived from measurements, such as haptic rendering, the drift becomes apparent.

The computational friction model proposed does not drift and provides several advantages for implementation. It is autonomous, and so suitable for event-based interfaces which may have nonuniform sampling. It is robust to noise, especially because it depends only on position. It is discrete in formulation, and so directly implementable by computer. It is extensible to $2 \mathrm{D}$ and 3D motions. Its parameters can be interpreted in terms of a surface deformation model, and it shows four regimes of behavior that are observed in physical systems.

The choice of a friction model depends on the application [2]. Here, the 
much simplified candidates for $\alpha(z)$ yield a variety of behaviors which are suitable for haptics. Various choices affect the haptic perception, so the question of perceptual relevance arises. For other applications, more direct association of the proposed friction model with tribophysics is needed, as is the introduction of dynamic friction effects. These are the subjects of continuing work.

\section{Acknowledgments}

The first author would like to thank McGill University for granting a sabbatical leave which made this research possible, and IRCAM, Paris, for generously hosting him. Thanks are due to X. Rodet and S. Tassart for many discussions on nonlinear oscillators. Special thanks to Joseph "Butch" Rovan, electronic music composer and performer for daring to use the reversal detector described in Section 3.1 during a public performance.

Funding for this research was also provided by the project "Core Issues in Haptic Interfaces for Virtual Environments and Communications" supported by IRIS (Phase 3), the Institute for Robotics and Intelligent Systems part of Canada's National Centers of Excellence program (NCE), and an operating grant "High Performance Robotic Devices" from NSERC, the National Science and Engineering Council of Canada.

\section{References}

[1] Hayward V., Astley O. R. 1996. Performance measures for haptic interfaces. In: Giralt G., Hirzinger G., Eds., 1996 Robotics Research: The 7th International Symposium, Springer Verlag. pp. 195-207.

[2] Armstrong-Helouvry B., Dupont P., and Canudas De Wit C., 1994. Survey of models, analysis tools and compensation methods for the control of machines with friction. Automatica. 30(7):1083-1138.

[3] Polycarpou A., Soom A. 1992. Transitions between Sticking and Slipping. In: Ibrahim R. A., Soom, A., Eds., 1992. Proc. Friction Induced Vibration, Chatter, Squeal, and Chaos, ASME Winter Annual Meeting, Anaheim, DE-Vol. 49, New York: ASME, pp. 139-48.

[4] Dahl, P. R. 1968. A solid friction model. TOR-158(3107-18). The Aerospace Corporation, El-Secundo, California.

[5] Karnopp D. 1985. Computer Simulation of stick slip friction in mechanical dynamic systems. ASME J. of Dyn. Systems, Meas. and Control. 107:100-103.

[6] Salcudean S. E., Vlaar T. D. 1994. On the emulation of stiff walls and static friction with a magnetically levitated input/output device. Proc. ASME Dynamic Systems and Control Division, DSC-Vol. 55-1, New York: ASME, pp.303-310.

[7] Haessig D. A., Friedland B. 1991. On the modeling and simulation of friction. ASME J. of Dyn. Systems, Meas. and Control. 113:354-362.

[8] Chen, J., DiMattia, C., Taylor II, R. M., Falvo, M., Thiansathon, P., Superfine, R. 1997. Sticking to the point: A friction and adhesion model for simulated surfaces. Prod. ASME Dynamics Systems and Control Division, DSC-Vol. 61, pp. 167-171.

[9] Dahl, P. R. 1976. Solid friction damping of mechanical vibrations. AIAA J., 14(2): $1675-82$.

[10] Canudas de Witt C., Olsson H., Aström K. J. 1995. A new model for control of systems with friction. IEEE T. on Automatic Control. 40(3):419-425. 